Revista Brasileira de Odontologia Legal - RBOL

Odontología Forense

\title{
ANÁLISIS DEL COMPORTAMIENTO DE LOS TEJIDOS DENTALES $Y$ MATERIALES DE OBTURACIÓN ENDODÓNCICOS SOMETIDOS A ALTAS TEMPERATURAS CON FINES FORENSES
}

\section{Dental tissues and endodontic obturation materials behavior analysis when submitted to high temperatures with forensic purposes}

Tamara Frontanilla RECALDE*1, Paulo Eduardo Miamoto DIAS ${ }^{2}$, Juan Carlos Zárate RODRÍGUEZ ${ }^{3}$, María Cristina González ESCOBAR ${ }^{4}$, Marcelo Costa PERDOMO ${ }^{5}$

\footnotetext{
${ }^{1}$ Doctor en Odontología. Universidad Autónoma del Paraguay. Académica en curso de Especialización en Odontología

${ }^{2}$ Legal Universidad de São Paulo - Ribeirão Preto.

${ }^{3}$ Doctor en Odontología Legal y Forense, Universidade de São Paulo. Coordinador de la ONG Equipe Brasileira de Antropologia Forense e Odontologia Legal - Ebrafol.

${ }^{4}$ Doctor en Odontología, Universidad del Norte, Asunción Paraguay. Presidente de la Sociedad Paraguaya de Odontología Legal y Forense. Jefe del Departamento de Criminalística de la Policía Nacional del Paraguay.

${ }^{5}$ Doctora en Odontología, Universidad Nacional de Asunción.

Doctor en Odontología, Univesidad Autónoma del Paraguay. Presidente de la Sociedad Paraguaya de Microscopía.
}

Informação sobre artigo

Recebido: 15 Jun 2015

Aceito em: 20 Jul 2015

\author{
Autor para correspondência \\ Tamara Frontanilla Recalde \\ Santiago casi Comandante Molas 1245 \\ Asunción, Paraguay \\ Email: tfronta@gmail.com
}

\section{RESUMEN}

Objetivo: Describir el comportamiento de los materiales de obturación y tejidos dentales sometidos a altas temperaturas con el fin de valorar la utilización de los mismos como parámetro de referencia en la estimación de la temperatura. Materiales y métodos: Para el análisis macroscópico, fueron utilizados 72 dientes humanos (24 anteriores, 24 premolares y 24 molares) endodonciados por la técnica escalonada, obturados por la técnica de condensación lateral, y posteriormente radiografiados. Para el análisis microscópico fueron utilizados 3 premolares. Se sumergieron grupos con tres dientes en bloques confeccionados con material de revestimiento refractario. Posteriormente los mismos fueron sometidos a altas temperaturas en un rango de $200^{\circ} \mathrm{C}$ a $800^{\circ} \mathrm{C}$, con intervalos de $200^{\circ} \mathrm{C}$. Alcanzada la temperatura propuesta, los bloques fueron retirados del horno. Los dientes fueron sumergidos en resina acrílica y 
cortados. Se observaron el estado de los tejidos dentarios así también los materiales de obturación y fueron comparados radiográfica, macroscópica y microscópicamente entre las diferentes temperaturas. Resultados: A cada temperatura, se observaron cambios significativos en los tejidos dentales y materiales de obturación, sobre todo en la coloración, los cuales a medida que aumenta la temperatura de exposición son mas relevantes, y esto facilita considerablemente la estimación de la temperatura a la cual fue expuesta. Radiográficamente, no se observan cambios significativos a los 200 y $400^{\circ} \mathrm{C}$ sin embargo a los 600 y $800^{\circ} \mathrm{C}$ se observan cambios en cuanto a la radiodensidad. Conclusión: los cambios experimentados constituyen una herramienta en procesos de estimación de la temperatura de exposición de restos mortales.

\section{PALABRAS CLAVES}

Calor, obturación del conducto radicular, fracturas de los dientes, Antropología Forense, Odontología Forense.

\section{INTRODUCCIÓN}

La aplicación de la Odontología Forense en procesos de identificación ha sido utilizada hace ya varios años, sin embargo, en la actualidad, ha cobrado una mayor importancia, debido a los avances tecnológicos, ya que los accidentes cobran más víctimas y las dejan menos reconocibles: incendios, explosiones dejan cuerpos mutilados y despedazados; sumado a ello, con la modernidad el hombre sufre nuevas formas de muerte en accidentes aéreos, automovilísticos e industriales entre otros en los cuales, en muchos casos, los cadáveres y restos humanos son afectados por la acción del fuego, por el cual quedan quemados, incinerados o calcinados, con lo que se dificulta la identificación ${ }^{1,2}$.

La individualidad de la dentición humana, así como la elevada resistencia ante la acción de grandes temperaturas, que caracteriza las piezas dentarias y los biomateriales odontológicos, le confieren a la Odontología Forense un papel determinante en la identificación médico legal de las víctimas de un desastre masivo ${ }^{3,4}$. Además de la información que se obtiene comparando los registros ante-mortem con los recogidos postmortem, también pueden aplicarse modernos métodos de análisis forense como el perfil de ADN a partir de especímenes obtenidos de la zona predentina y la pulpa dental $^{5,6}$. 
Considerando la creciente demanda de tratamientos endodóncicos en el Paraguay, tomando como referencia los casos tratados en la Universidad Autónoma del Paraguay, donde en promedio son realizadas 1200 endodoncias anualmente. Teniendo en cuenta que existen 23 Facultades de Odontología en el país, hace referencia que el porcentaje poblacional con ésta terapéutica pueda ser elevado, lo cual lleva a entender que en grades catástrofes existe un altísimo porcentaje de víctimas podrían identificarse. Por lo tanto, el conocimiento del comportamiento de estos tejidos y materiales es relevante a la Odontología Forense.

\section{MATERIALES Y MÉTODOS}

Se condujo un estudio experimental ex vivo, acerca del comportamiento de los tejidos dentales (esmalte, dentina $y$ cemento) y materiales de obturación endodóncicos [conos de gutapercha, cemento Sealer $26^{\circledR}$ (Dentsply, York, PA, EE.UU.)] sometidos a la acción del calor.

Setenta y cinco dientes humanos (25 dientes anteriores, 25 premolares y 25 molares) fueron endodonciados por la técnica escalonada, obturados con gutapercha y cemento Sealer $26^{\circledR}$ por la técnica de condensación lateral. Dichos dientes fueron radiografiados posteriormente. Para el análisis macroscópico, fueron seleccionados 72 dientes humanos, mientras que para el análisis microscópico fueron utilizados tres dientes humanos.

Para la exposición térmica, se sumergieron grupos de tres dientes en bloques confeccionados con material de revestimiento refractario [1700 ${ }^{\circledR}$ (Talmax, Curitiba, PR, Brasil)] lo cual simularía la acción de los tejidos periféricos que los aísla de la acción directa del calor por algún tiempo. Posteriormente los mismos fueron puestos en un horno y sometidos a altas temperaturas en un rango de $200^{\circ} \mathrm{C}$ a $800^{\circ} \mathrm{C}$, en intervalos de $200^{\circ} \mathrm{C}$. A cada intervalo, grupos de bloques fueron sacados para análisis. Después de removidos del revestimiento, los dientes fueron cortados transversalmente utilizando discos, exponiendo así el contenido del conducto radicular. Se observaron seguidamente el estado de los tejidos dentarios (esmalte, dentina y 
cemento) así también los materiales de microscópicas y radiográficas de los tejidos dentales duros $y$ obturadores endodóncicos entre las diferentes temperaturas.

\section{RESULTADOS}

Las alteraciones de los tejidos duros son descritas en la Tabla $1 \mathrm{y}$ observadas en las Figuras 1 a 7 . Las alteraciones de los materiales de obturación son descritas en la Tabla 2 y observadas en las Figuras 4 a 7. Los cambios radiográficos de los especímenes sometidos al calor se encuentran en la Tabla 3 y observadas en la Figuras 8 y 9 , mientras las alteraciones microscópicas son descritas en la Tabla 4 y observadas en la Figuras 10 y 11.

Tabla 1 - Cambios estructurales de los tejidos dentales sometidos a altas temperaturas.

\section{${ }^{\circ} \mathrm{C} \quad$ Cambios}

200 Perdida de brillo del esmalte, ligero oscurecimiento del cemento

Oscurecimiento del esmalte. Grietas y fisuras a nivel cervical

La dentina se presenta de color negro

El cemento se observa de un color marrón oscuro

En algunos casos se produjo el estallido de la corona a nivel cervical y separación de la porción coronaria de la porción radicular.

Oscurecimiento del esmalte, adquiere un color grisáceo

600 Dentina de color negro

Cemento presenta una coloración grisácea

Esmalte de coloración gris claro veces imposible diferenciar los tejidos unos de otros. 
Tabla 2 - Cambios estructurales de los materiales de obturación endodóncicos sometidos a altas temperaturas

\begin{tabular}{ll}
${ }^{\circ} \mathbf{C} \quad$ Cambios \\
\hline $200 \quad$ Sin cambios significativos. \\
& $\begin{array}{l}\text { Ligero desprendimiento del material obturador con respecto a las paredes } \\
\text { del conducto }\end{array}$ \\
$400 \quad \begin{array}{l}\text { La gutapercha se vuelve más opaca, presentando zonas blancas } \\
\text { entrelazadas }\end{array}$ \\
$600 \quad \begin{array}{l}\text { La gutapercha aparece incinerada con una coloración blanco tiza } \\
800 \quad \begin{array}{l}\text { La gutapercha se encuentra totalmente incinerada siendo imposible } \\
\text { diferenciarla del tejido dentinario. }\end{array}\end{array}$ \\
\hline
\end{tabular}

Tabla 3 - Cambios radiográficos de los tejidos dentales y materiales de obturación endodóncicos sometidos a altas temperaturas.

\begin{tabular}{ll}
\hline${ }^{\circ} \mathbf{C}$ & Cambios \\
\hline 200 & Sin cambios \\
$400 \quad \begin{array}{l}\text { Sin cambios significativos. Zonas radiolúcidas a nivel del esmalte y la } \\
\text { dentina compatibles con fracturas post-incineración de los tejidos } \\
\text { dentarios apreciables macroscópicamente }\end{array}$ \\
$\begin{array}{l}\text { Ligeras áreas radiolúcidas (poros) en la zona ocupada por los } \\
\text { materiales de obturación endodóncicos, compatibles probablemente } \\
\text { con la incineración de los mismos. Zonas radiolúcidas a nivel del } \\
\text { esmalte y la dentina compatibles con fracturas post-incineración de los } \\
\text { tejidos dentarios apreciables macroscopicamente }\end{array}$ \\
$\begin{array}{l}\text { Las áreas radiolúcidas en la zona de los materiales de obturación } \\
\text { endodóncicos son más evidentes. }\end{array}$ \\
\hline
\end{tabular}


Tabla 4 - Cambios microscópicos de los tejidos dentales y materiales de obturación endodóncicos sometidos a altas temperaturas.

\section{${ }^{\circ} \mathrm{C}$ Cambios}

Destrucción total de la región pulpar. En el tejido dentinario podemos diferenciar dos zonas, una de mayor destrucción donde hay ausencia

200 de la morfología normal y otra de mayor contenido de sustancia orgánica y los procesos odontobásticos pueden apreciarse ligeramente.

Destrucción total de la morfología normal de la zona dentinaria.

400 Además, un entramado no compatible con las características morfológicas normales.

600 No se logró la observación.

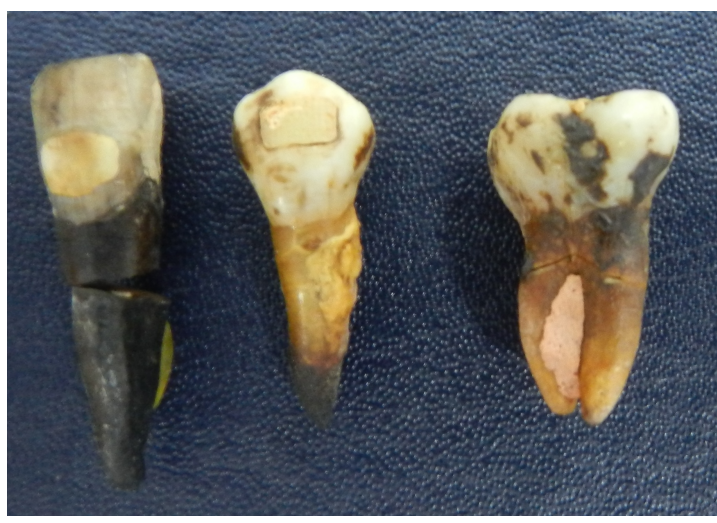

Figura 1 - Cambios estructurales de los tejidos dentales a $400{ }^{\circ} \mathrm{C}$.

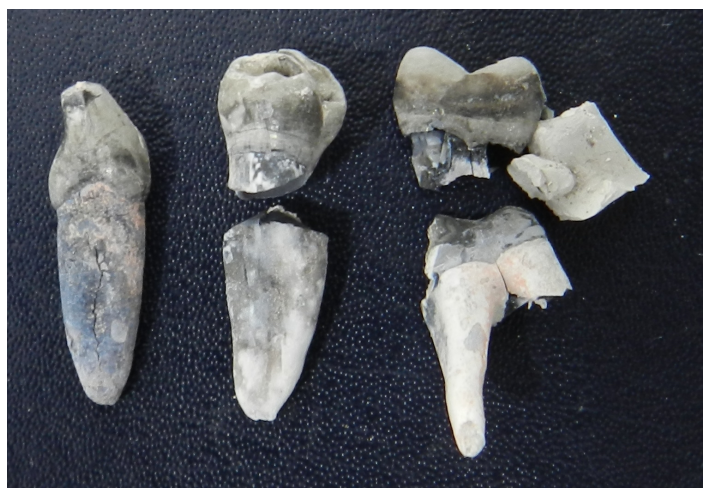

Figura 2 - Cambios estructurales de los tejidos dentales a $600^{\circ} \mathrm{C}$.

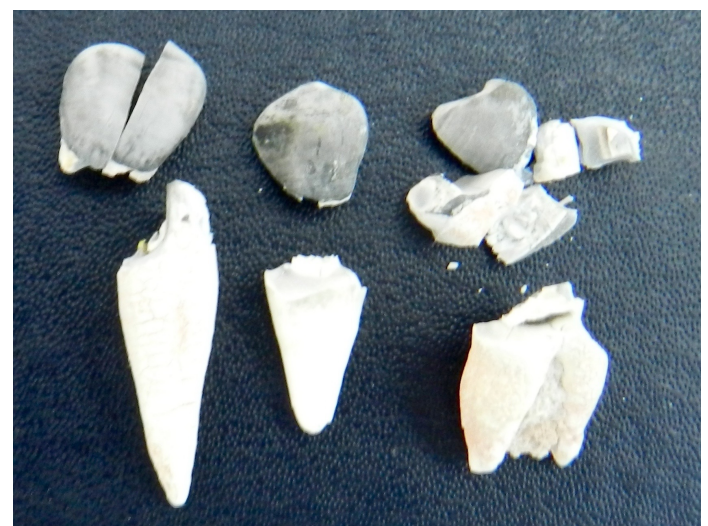

Figura 3 - Cambios estructurales de los tejidos dentales a $800{ }^{\circ} \mathrm{C}$.

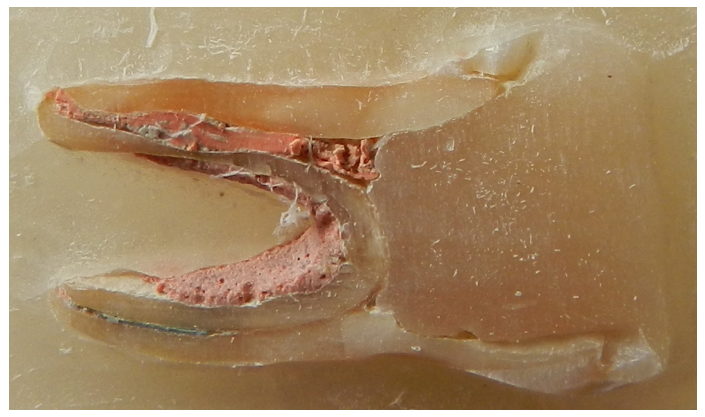

Figura 4 - Cambios estructurales de los materiales de obturación endodóncicos a $200{ }^{\circ} \mathrm{C}$. 


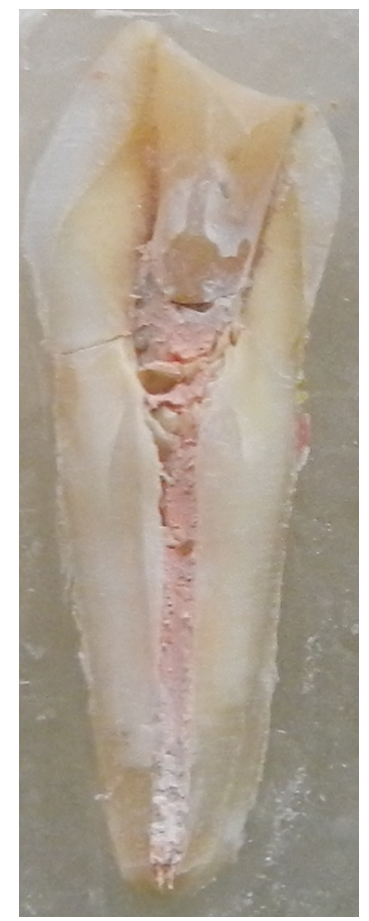

Figura 5 - Cambios estructurales de los materiales de obturación endodóncicos a $400{ }^{\circ} \mathrm{C}$.

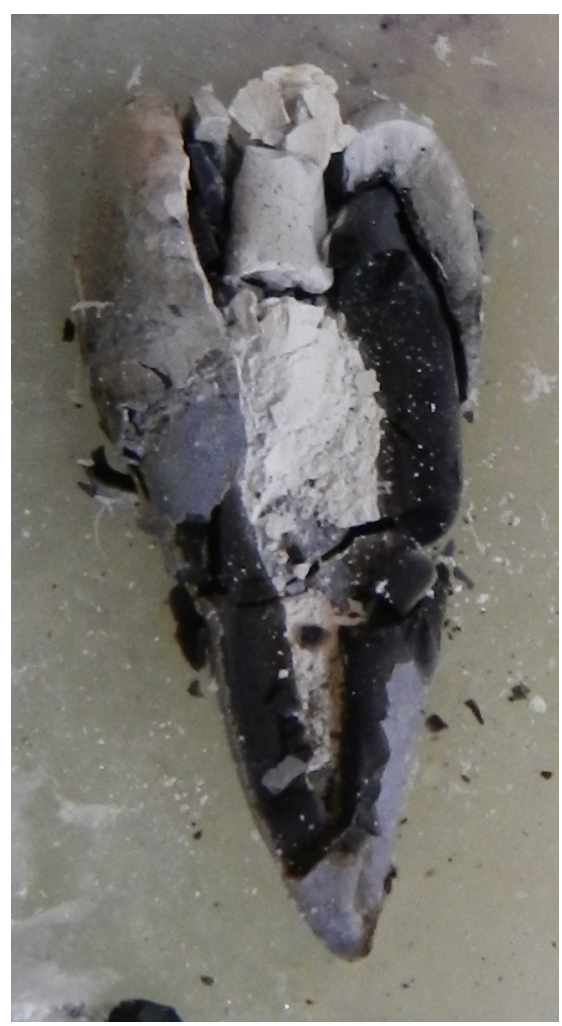

Figura 6 - Cambios estructurales de los materiales de obturación endodóncicos a $600{ }^{\circ} \mathrm{C}$.

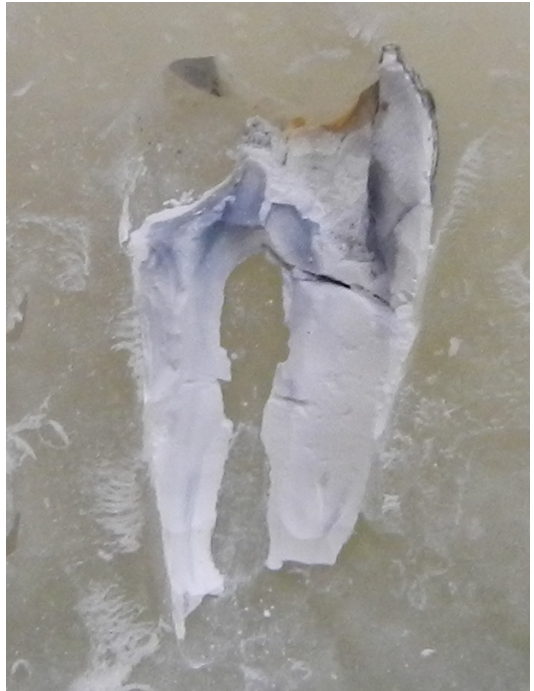

Figura 7 - Cambios estructurales de los materiales de obturación endodóncicos a $800{ }^{\circ} \mathrm{C}$.

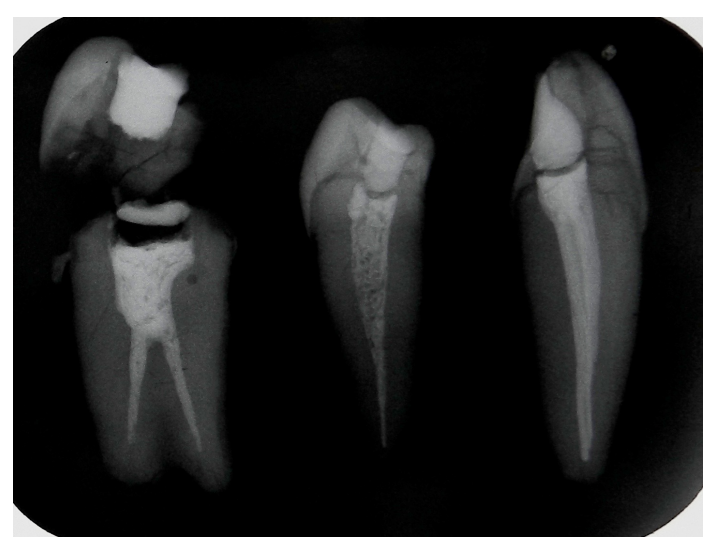

Figura 8 - Cambios radiográficos de los tejidos dentales y materiales de obturación endodóncicos a $600{ }^{\circ} \mathrm{C}$.

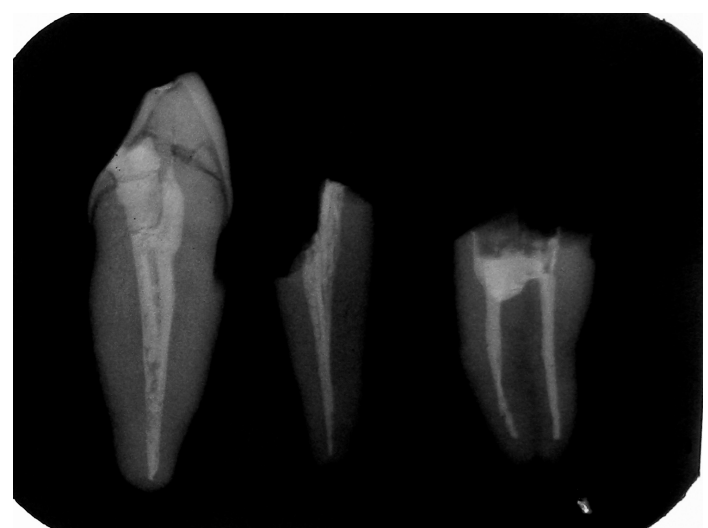

Figura 9 - Cambios radiográficos de los tejidos dentales y materiales de obturación endodóncicos a $800{ }^{\circ} \mathrm{C}$. 


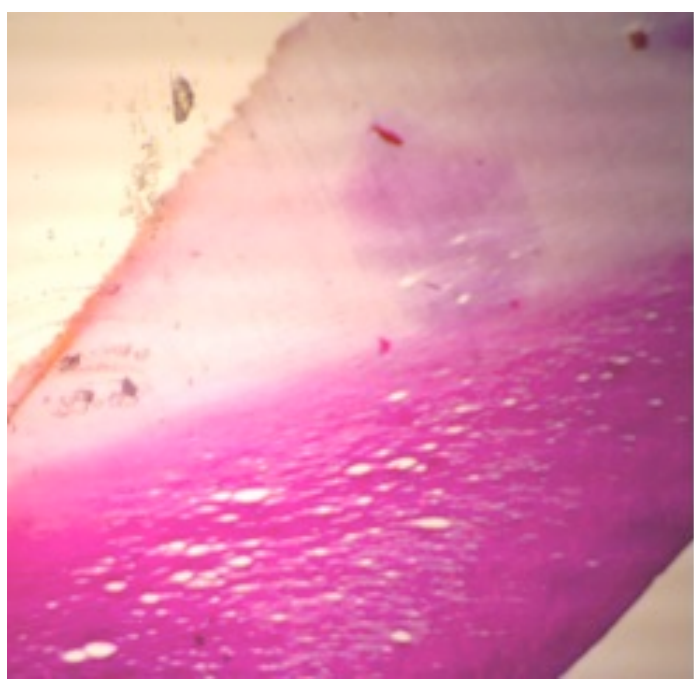

Figura 10 - Cambios microscópicos de los tejidos dentales y materiales de obturación endodóncicos a $200^{\circ} \mathrm{C}$.

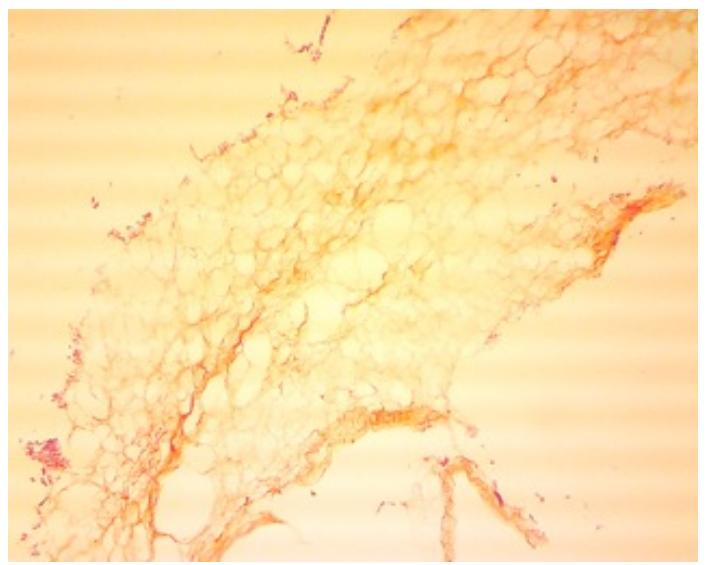

Figura 11 - Cambios microscópicos de los tejidos dentales y materiales de obturación endodóncicos a $400^{\circ} \mathrm{C}$.

Microscópicamente a $200^{\circ} \mathrm{C}$ se observa una destrucción total de la región pulpar. Con respecto a la dentina, podemos apreciar dos zonas diferenciadas, donde la primera refiere una mayor destrucción de tejido y la segunda porción, de menor destrucción y mayor cantidad de sustancia orgánica, probablemente porque la exposición al calor no se produce de forma uniforme.

Los resultados microscópicos son representativos pero no concluyentes, por lo cual se recomienda la realización de una nueva línea de investigación acerca del tema.

\section{DISCUSIÓN}

Este estudio ex vivo evidencia cómo los tejidos dentales y los materiales de obturación endodóncicos presentan comportamientos diferentes en cada rango de temperatura, sin embargo in vivo estos cambios pueden variar según factores extrínsecos como tiempo de exposición al ataque térmico, naturaleza de la causa del fuego, participación de sustancias combustibles, curva de elevación de la temperatura y sustancias empleadas para extinguir el fuego, etc. como lo indica también Moreno et al. ${ }^{7}$ en su estudio realizado.

$$
\text { Los tejidos dentales }
$$
específicamente esmalte, dentina y cemento presentan alteraciones significativas útiles en el proceso de estimación de la temperatura expuesta. 
Lo más relevante en este estudio, es que si bien todos los tejidos dentarios experimentan cambios a cada temperatura propuesta, el cemento dental mostró un cambio mucho más representativo y de fácil diferenciación macroscópica en las cuatro temperaturas propuestas.

Este cambio de coloración que experimenta el cemento dental a cada temperatura, es de interés extraordinario ya que con una observación macroscópica, se podría rápidamente estimar la temperatura de exposición de las piezas dentarias y aportar datos en un tiempo muy corto en casos de desastres en masa, sin necesidad de llevarlos a un estudio mas invasivo, realizando cortes en las piezas dentarias para exponer el contenido del conducto o la dentina que rodea al mismo, lo que alargaría el proceso de estimación de la temperatura de exposición.

En este estudio, se pudo observar un fenómeno característico: la fractura de la unión amelocementaria y separación de la corona, lo cual probablemente sea el resultado de la diferencia de estructura orgánica e inorgánica presente en cada tejido, muy similar a lo demostrado en estudios previos $^{7-10}$.

Se pudo observar que a partir de $200^{\circ} \mathrm{C}$ existe fractura $y$ en algunos casos una separación mínima del esmalte y de la dentina del margen cervical y que sin embargo partir de los $400^{\circ} \mathrm{C}$ la separación es completa a este nivel, dejando una división clara y característica entre corona-raíz. Estos resultados fueron semejantes a los obtenidos en investigaciones anteriores $^{7,11,12}$.

Radiográficamente

no existieron cambios significativos hasta los $600^{\circ} \mathrm{C}$, sin embargo a temperaturas mayores se logró observar zonas más radiolúcidas, pero manteniéndose el material obturador en el interior del conducto, un resultado similar al demostrado por Savio et al. ${ }^{13}$.

Los cambios que experimentan los materiales de obturación endodóncicos son de igual manera útiles en el procesos de estimación de la temperatura a la cual estuvo expuesta esa pieza dentaria ya que estos cambios son apreciables de manera macroscópica en cuanto a coloración y desadaptación marginal, resultados similares a los obtenidos 
por Moreno et $\mathrm{al}^{7}$. A pesar de esto, la ubicación intra canal de los materiales endodóncicos puede ser un obstáculo a su evaluación, en casos donde el calor no exponga a la superficie radicular.

\section{CONCLUSIÓN}

Las variaciones producidas en los tejidos dentarios y materiales de obturación endodóncicos, son apreciables macroscópicamente lo cual constituye una herramienta útil en los procesos de identificación en casos de cadáveres incinerados, y la estimación de la temperatura a la cual estuvo expuesta el cadáver. Cuando es posible su visualización, el cemento es el tejido dental más útil para esta labor, pues experimenta cambios muy distintos en su coloración.

\section{ABSTRACT}

Objective: To describe the behavior of endodontic obturation materials dental tissues subjected to high temperatures in order to assess their use as a parameter for forensic assessment of the temperature of burnt corpses. Materials and Methods: Seventy-two human teeth with endodontic treatment by the stepwise technique, sealed with gutta-percha and cement were selected for macroscopic analysis (24 anterior teeth, 24 premolars and 24 molars). Three human teeth (premolars) were selected for microscopic analysis. Groups with three teeth were submerged in blocks of dental casting. Next, they were exposed to high temperatures within a range from $200^{\circ} \mathrm{C}$ to $800^{\circ} \mathrm{C}$, at intervals of $200{ }^{\circ} \mathrm{C}$. At each temperature interval, part of the blocks was removed for analysis and radiographic examination. Teeth were further submerged in acrylic resin and cut with discs, thus exposing the radicular contents. The conditions of the dental tissues (enamel, dentin and cementum) and obturation materials were compared between different temperatures, both macroscopically and microscopically. A comparative radiographic analysis of the material was also conducted. Results: At each temperature range, significant alterations were observed, especially regarding coloration, which are more relevant as temperatures of exposition increase. The estimation of the temperature to which dental tissues and materials were exposed is considerably facilitated. In radiographic examination, significant changes are not observed at $200^{\circ} \mathrm{C}$ and $400^{\circ} \mathrm{C}$, nevertheless at $600^{\circ} \mathrm{C}$ and $800^{\circ} \mathrm{C}$ radiodensity alterations are observed. Conclusion: Alterations of dental tissues and obturation materials represent a valid tool for the assessment of the temperature to which human remains were exposed.

\section{KEYWORDS}

Hot temperature, root canal obturation, tooth fractures, Forensic Anthropology, Forensic Dentistry 


\section{REFERENCIAS}

1. Sweet D. Why a dentist for identification? Dent Clin North Am. 2001; 45(2):237-51.

2. Senn D, Weems R. Manual of Forensic Odontology, 5th ed. Colorado Springs: American Society of Forensic Odontology; 2013.

3. Clark DH. An analysis of the value of forensic odontology in ten mass disasters. Int Dent J. 1994; 44(3): 241-50.

4. Valenzuela A, Martin-de las Heras $\mathrm{S}$, Marques T, Exposito N, Bohoyo $\mathrm{J}$. The application of dental methods of identification to human burn victims in a mass disaster. Int J Legal Med. 2000; 113(4): 236-9.

5. Andersen $L$, Juhl $M$, Solheim $T$, Borrman H. Odontological identification of fire victimspotentialities and limitations. Int $\mathrm{J}$ Leg Med. 1995; 107(5): 229-34

6. Mazza A, Merlati G, Savio C, Fassina G, Menghini P, Danesino $P$. Observations on dental structures when placed in contact with acids: Experimental studies to aid identification processes. J Forensic Sci. 2005; 50: 406-10.

7. Moreno S, León M, Marín L, Moreno F. Comportamiento in vitro de los tejidos dentales y de algunos materiales de obturación dental sometidos a altas temperaturas con fines forenses. Colomb Med. 2008; 39(1)Supl 1: 28-46.

8. Yamamoto K, Ohtani $\mathrm{S}$, Kato $\mathrm{S}$, Sugimoto H, Miake K, Nakamura T. Morphological changes in human and animal enamel roots with heading -especially limits in temperature allowing discrimination between human and animal teeth. Bull Kanagawa Dent Coll. 1990; 18(1): 55-61.

9. Espina A, Barrios $F$, Ortega A, Mavaréz $M$, Espina $O$, Ferreira $J$. Cambios estructurales en los tejidos dentales duros por acción del fuego directo, según edad cronológica. Cien Odontol. 2004; 1(1): 38-51.

10. Bonavilla JD, Bush MA, Busha PJ, Pantera EA. Identification of incinerated root canal filling materials after exposure to high heat incineration. J. Forensic Sci. 2008; 53(2):412-8. $<$ doi:10.1111/j.15564029.2007.00653.x>

11. Moya V, Roldan B, Sánchez JA. Materiales dentales en la identificación. In: Odontología legal $y$ forense. Barcelona: Editorial Masson SA; 1994. p. 269-76.

12. Valdéz $Y$. Odontología legal y forense. In: Herazo B (ed.). Odontología Forense. Santa Fe de Bogotá: Ecoe Editores; 1995. p. 85111.

13. Savio C, Merlati G, Danesino P, Fassina G, Menghini. Radiographic evaluation of teeth subjected to high temperatures: Experimental study to aid identification processes. Forensic Sci Int. 2006; 158(2-3): 108-16. <doi:10.1016/j.forsciint.2005.05.003 $>$ 